\title{
Origen, localización y evolución de los panares del municipio de Gistaín
}

\author{
Carlos Fernández Piñar
}

PALABRAS CLAVE: Pirineos, sistemas agrícolas, panares, comunales.

\section{CÓDIGOS JEL: N53, O13, Q15, R14.}

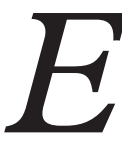

objetivo de este trabajo es abordar el estudio de un particular sistema agropecuario, característico del ámbito pirenaico, a partir del ejemplo concreto de un municipio ubicado en uno de los altos valles del Pirineo oscense. La elección de este municipio, Gistaín, viene dada por ser uno de los enclaves en que este tipo de práctica tuvo un mejor grado de conservación hasta tiempos muy recientes, llegando a alcanzar la década de los años ochenta del siglo pasado, mientras en otros valles desapareció mucho antes.

En primer lugar, se hace una aproximación a este modelo de explotación, denominado panar, desde una perspectiva general y a la luz de los pocos trabajos que han tratado el tema, para a continuación centrar el foco en el área de estudio. El municipio de Gistaín se analiza, en su configuración de mediados del siglo XIX, a partir de tres fuentes fundamentales: el estudio de campo sobre el terreno, la tradición oral recogida entre los habitantes de más edad del municipio y el registro documental, fundamentalmente el amillaramiento de 1863. Con los datos aportados y la consulta de algunos documentos antiguos conservados en el archivo municipal de Gistain, en su mayor parte de los siglos XVI y XVII, se proponen en las conclusiones algunas ideas acerca del origen y la evolución de estos espacios. 


\section{Origin, location and evolution of the panares in the Gistaín municipality}

\section{KEYWORDS: Pyrenees, agricultural systems, panares, commons.}

\section{JEL CODES: N53, O13, Q15, R14.}

he aim of this paper is to study an agricultural system typical of the Pyrenean
area, based on the example of Gistain, a municipality located in a high val-
ley of the Aragonese Pyrenees. In this enclave, the panar system endured until the 1980s, while in other valleys it disappeared much earlier.

First, the panar model is approached from a general perspective using the limited literature available on the subject. The focus then shifts to the study area and the Gistain municipality is analysed in its mid-nineteenth-century configuration, based on three fundamental sources: a field study of the land, oral tradition collected from the older inhabitants and documentary records, mainly the amillaramiento of 1863. Some older documents, mainly from the sixteenth and seventeenth centuries, were also consulted in the Municipal Archive. In the conclusions, some ideas are provided about the origin and evolution of these spaces. 


\section{INTRODUCCIÓN}

En el Pirineo aragonés existieron hasta hace pocas décadas prácticas comunitarias que permitieron, por una restricción del derecho de propiedad, mantener el dominio pastoral ante la expansión de los cultivos (Daumas, 1961: 16). Una de estas prácticas fueron los denominados panares. Este trabajo aborda el estudio de este particular sistema agropecuario como parte integrante de un paisaje cultural $^{1}$. Este enfoque pone en relación actividades económicas, aspectos históricos y sociales, y formas de apropiación y construcción del territorio.

La primera parte del artículo está orientada a definir qué es un panar, sus características, su organización a lo largo del ciclo anual y su distribución en el ámbito del Pirineo central a partir de los trabajos que han tratado estos espacios con anterioridad desde la perspectiva de la geografía, la ecología, o de su valor paisajístico. La segunda parte centra el foco en los panares del municipio de Gistaín. Los objetivos planteados son delimitar su localización dentro del territorio municipal, estudiar su forma de organización y el papel que desempeñaron dentro del sistema agropecuario local, entender su evolución y analizar su impronta en la configuración del paisaje actual. El estudio está centrado fundamentalmente en el marco cronológico de mitad del siglo xIX a mediados del siglo xx, porque son muy escasas las referencias documentales a periodos anteriores y la tradición oral no permite retroceder mucho más atrás. Aun así, se busca poder situar cronológicamente el origen de este modo de explotación a partir de algunas referencias dispersas en documentos más antiguos y de los datos de recientes estudios paleoambientales. El trabajo está basado en tres fuentes principales: el trabajo de campo, con el reconocimiento de las parcelas que constituyeron los panares; la tradición oral recogida de los habitantes de más edad de la población, que aún llegaron a utilizarlos; y el registro documental, sobre todo el primer amillaramiento del año 1863, a partir del cual, con la ayuda de los informantes locales, ha sido posible reconstruir la estructura de la propiedad de las distintas casas de Gistaín a mediados del siglo XIX.

\section{LOS PANARES EN EL CONTEXTO PIRENAICO}

No existe por el momento ningún estudio monográfico sobre este tipo de explotación. Las referencias son tangenciales en trabajos como los estudios del geógrafo francés Max Dau-

1. Según la Convención del Patrimonio Mundial de la UNESCO, los Paisajes Culturales son bienes culturales que representan obras conjuntas del hombre y la naturaleza que ilustran la evolución de la sociedad humana, bajo la influencia de las limitaciones físicas y/o las posibilidades de su medio 
mas $(1961,1976)$ sobre la economía rural de las comarcas de Sobrarbe y Ribagorza. Cabe destacar también la contribución de Fernando Biarge, que ha insistido en numerosas publicaciones sobre el valor paisajístico y cultural de los panares (Biarge \& Biarge, 2000: 3239; Biarge, 2009: 222-231). Diversos trabajos realizados desde el Instituto Pirenaico de Ecología también los han tratado desde la óptica de los usos del suelo, la botánica de los pastos y la agricultura de montaña (Balcells \& Creus-Novau, 1986; Lasanta, 1989: 89; García-Ruiz et al., 2015). Por otro lado, se han descrito los panares incluidos dentro del Bien Pirineos-Monte Perdido, inscrito en la lista del Patrimonio Mundial por la UNESCO, en un documento todavía inédito (Pardinilla, 2013).

En el Pirineo central, como en otras áreas de montaña, la organización de los espacios productivos estaba estratificada en diferentes escalones, diferenciados por su altitud y su aprovechamiento. Mientras en las proximidades de los núcleos de población los usos agrarios eran dominantes, a medida que nos alejamos y ganamos altura los usos ganaderos tomaban mayor importancia, para acabar siendo los únicos en el área ocupada por los pastos de altura, las estivas y los puertos.

Los panares constituían un espacio intermedio entre estos dos polos, con un uso mixto agrario y ganadero en el que se alternaban el cultivo de cereales para autoconsumo ${ }^{2}$ y el uso como pastos para el ganado en distintos momentos del ciclo anual. Estaban formados por campos de labor situados distantes del pueblo y en altitud, generalmente entre los 1300 y los 1800 metros, en los que se producía cereal, sobre todo centeno, porque se adaptaba mejor a las bajas temperaturas y a los suelos pobres, y también patatas desde su introducción a mediados del siglo XviII. Eran propios de los pueblos con términos municipales de altitudes medias considerables y relieve muy accidentado, donde las escasas posibilidades de aprovechamiento agrícola obligaban a aumentar la superficie dedicada a los cultivos y a poner en producción laderas alejadas en las que por sus condiciones de orientación, suelos o disminución de la pendiente aparecía la más mínima oportunidad (García-Ruiz, 1988: 12). En particular, es recurrente la existencia de este tipo de campos sobre los depósitos sedimentarios correspondientes a las morrenas laterales de los antiguos glaciares de los altos valles (Daumas, 1976: 251; Fillat \& Villar, 1983; Lasanta, 1989: 89).

ambiente natural y de las fuerzas sociales, económicas y culturales sucesivas, tanto internas como externas. (Comité intergouvernemental pour la protection, 2008: 47).

2. La producción de cereales en estos valles pirenaicos fue casi siempre insuficiente para cubrir las necesidades de la población. A finales del siglo XviII el valle de Benasque podía autoabastecerse de cereal, pero en el valle de Broto faltaba para un cuarto del año, en el de Gistaín para un tercio, en el valle de Vió y el valle de Tena para la mitad, y en Bielsa para ocho meses (BuIL, 1997). 
El sistema de cultivo empleado en los panares era de año y vez, con un ciclo bienal en el que se cultivaba la tierra un año y se dejaba en barbecho el siguiente. Esta regla respondía también a un factor puramente climático. Debido al ciclo vegetativo extremadamente lento, como resultado de las bajas temperaturas, se sembraba el centeno en el mes de agosto y se recogía la cosecha a finales del mismo mes o durante septiembre del año siguiente, demasiado tarde para preparar el suelo para una nueva cosecha (Daumas, 1976: 324-325; Balcells \& Creus-Novau, 1986: 166; Lasanta, 1989: 55, 89)³. Por esta razón, los panares formaban dos conjuntos, situados en diferentes zonas del término de un pueblo, de forma que se cultivaban siguiendo un régimen alternativo obligatorio entre ellos. El panar que se encontraba en barbecho era abonado por los rebaños ovinos aproximadamente desde mediados de mayo hasta finales de julio, aprovechando su estancia en los pastos de tránsito, también denominados bajantes o femallas. Estos pastos, en contacto con las fincas particulares de los panares, constituían un paso intermedio en el periplo del ganado desde los pastos de invernada hasta los pastos estivales, en los puertos. Se hacía pernoctar a los animales encerrados en corrales móviles ${ }^{4}$ que se cambiaban de lugar cada día. Por ello, las parcelas incluidas dentro de los panares carecían de cierres o divisiones. Para facilitar el tránsito de los animales, las laderas que ocupaban eran aterrazadas por medio de suaves taludes herbosos, que constituyen uno de sus más destacados rasgos paisajísticos, y no mediante muros ${ }^{5}$.

Del mismo modo, a la hora de bajar de los puertos, los rebaños acudían al otro $p a-$ nar, para entonces recién cosechado, y aprovechaban como pasto el rastrojo. Así, el cultivo del centeno cerraba su ciclo productivo, usando alternativamente los panares. Este es el caso de localidades como Sahún, donde alternaban el panar de Llisat y el panar de Sila, o el de Aneto, con los panares de la Menada y Lestuy (Daumas, 1961: 17-18).

Esta alternancia en el cultivo por zonas de campos, acordada entre todos los vecinos para usar el barbecho y el rastrojo como pastos comunales, era común en muchas áreas pirenaicas y prepirenaicas, y recibía el nombre de añadas u hojas (Lasanta, 1989: 72). Según Daumas (1961: 16), las rotaciones regladas obligatorias se extendían por Navarra y

3. Este ciclo largo del centeno de trece meses contrasta con el del mismo cultivo en algunos valles alpinos, donde se empleaba un ciclo corto con siembra en primavera, a pesar de la mucho mayor latitud (Balcells \& Creus-Novau, 1986).

4. Reciben diferentes nombres según los valles. Así, en Gistaín se denominan barandau, y baranato en el valle de Bielsa. Ambos proceden del vocablo barana, que denomina cada uno de los segmentos que conforman el corral. En otros valles oscenses este elemento se llama cleta, y el corral cletau.

5. La distribución espacial de este tipo de bancales en el Pirineo aragonés viene a coincidir con la de los panares, siendo mucho menos frecuentes en los valles occidentales (LASANTA, GARCÍA-RUIZ \& ORTIGOSA, 1994). 
por el Pirineo aragonés al oeste del río Cinca, sobre todo en las depresiones intrapirenaicas ocupadas por grandes extensiones homogéneas, y eran raras en cambio en los valles aragoneses orientales del Sobrarbe y la Ribagorza, con parcelaciones mucho más fragmentadas, salvo el caso de los panares de los valles más altos (Daumas, 1976: 250). Por ejemplo, en Escartín, en el Sobrepuerto, existen sendas colinas situadas a cada uno de los lados del pueblo, que se cultivaban según este sistema de añadas. Lo mismo sucedía en los pueblos de La Solana de Burgasé, donde mediante un acuerdo tácito se agrupaban los trigales de cada año a un lado del pueblo y los barbechos al otro, lo que facilitaba el pastoreo de los rebaños lejos del sembrado (Buisán, 2004: 132). En Lamiana se cultivaba cada año una vertiente del barranco de la Fuen. En Bies los campos quedaban divididos en dos zonas, la añada de Arriba y la añada de Abajo, cultivadas en años alternos (Lasaosa \& Ortega, 2003: 204). En estos casos la alternancia obligatoria parece responder a un acuerdo entre vecinos propietarios de parcelas colindantes de pequeño tamaño y difícil acceso, para evitar los daños en los cultivos y las consiguientes disputas que se producirían en caso de efectuarse los trabajos agrícolas de forma desordenada (Daumas, 1976: 250), pero no reciben el nombre de panares. Esto parece indicar que el término se reservaba para los campos más alejados de los núcleos de población y ya en contacto con los pastos comunales.

Actualmente, en muchos lugares, el vocablo panar se refiere a cualquier campo dedicado a la producción de cereales ${ }^{6}$, independientemente de la distancia al núcleo. En la documentación del siglo XIV parece designar genéricamente a los campos cultivados (Utrilla, Laliena \& Navarro, 2005). También parece haberse perdido en muchos casos la regulación comunal del aprovechamiento de los panares como pastos, al consumir cada propietario lo suyo. Pero en su acepción estricta podríamos considerar que un panar es un espacio en el que se cultivaba cereal, situado a una cierta distancia del núcleo de población, que podía variar entre apenas un cuarto de hora y hasta cuatro horas de camino, siempre en discontinuidad física con los campos de cultivo primarios de alrededor del pueblo, existiendo espacio comunal entre ambos, y sobre el que se imponían restricciones al uso y aprovechamiento particular para compaginar los ritmos pastoriles comunales con los cultivos de cada casa. Visualmente los panares se caracterizan por la continuidad de sus parcelas, separadas tan solo por taludes de hierba, y la existencia de un número variable de pequeñas construcciones accesorias (cabañas). Las reglas que los regulaban eran consuetudinarias y no aparecían casi nunca formuladas en un documento ${ }^{7}$. En ellas se

6. Así aparece reflejado en muchos diccionarios y glosarios (BUISÁN, 2004: 231; BLAS \& Romanos, 2008: 401).

7. En cambio, en el caso de los llamados panificados del valle del Roncal, sí se conservan las normas de uso escritas en las Ordenanzas (Alli, 1989: 231). 
aprecia la fuerza de la comunidad y el papel preponderante de la ganadería sobre la agricultura (Biarge \& Biarge, 2000: 37).

Esta organización reglada de los aprovechamientos podía alcanzar un grado de complejidad considerable, que variaba de un pueblo a otro en función del número y la localización concreta de sus panares. Es este un aspecto que está aún por estudiar en muchos municipios. Algunos disponían de un único panar, por lo que habría que ver cómo era su régimen de explotación, ya que la alternancia de panares no era posible. En el caso del panar de Diera, en Espierba, se dividía su extensión en dos partes, de acá y de diyá, que se alternaban. En realidad, en esta localidad existían otros panares, pero de menor extensión y calidad, repartidos en distintos lugares y algunos de ellos compartidos con otros núcleos del mismo municipio de Bielsa. Su organización temporal ya no se recuerda ${ }^{8}$.

En muchos otros lugares los panares eran numerosos, como en Cerler ${ }^{9}$ o en San Juan de Plan, donde se alternaban en dos grupos, procurando siempre que el número de campos de uno y otro fuera más o menos parecido.

Los panares nos plantean varias cuestiones por resolver. La primera es dónde situar cronológicamente su origen. Parece ser que, en un primer momento, los panares ocuparon suelo comunal, sin que existiera propiedad sobre el terreno, sino tan solo derecho sobre la cosecha (Pascua, 2012: 114). No había propiedad tampoco sobre el aprovechamiento del rastrojo, que era comunal (Fillat, 1980a). A partir de este inicio se pudo ir consolidando la propiedad de las parcelas, pero manteniendo la servidumbre como zona de pasto comunal cuando no estaban cultivadas.

Daumas (1976: 123) achaca la expansión de los cultivos, ya sea sobre pendientes escarpadas, a base de aterrazamientos, como en zonas altas, en contacto con las estivas y al límite de las posibilidades climáticas, al crecimiento demográfico de los siglos XVIII y XIX, lo que puede sugerir que el origen de los panares se encuentre en este momento. Sin embargo, existen argumentos para situarlo mucho antes. Otros autores (Bielza de Ory et al., 1986) han señalado un incremento de la trashumancia de largo recorrido desde el principio del siglo XIV, con aumento de la cabaña ovina y, por tanto, de la necesidad de pastos, todo ello coincidiendo con un auge demográfico y un incremento de la presión agrícola sobre el territorio. En el valle de Tena está documentado un rápido crecimiento

\section{Información oral de Ángel Luis Saludas, Casa Barré de Espierba.}

9. En Cerler se cuentan hasta siete panares: La Rueda, Paluenga, Guariz, Ubiergo, Clot, Micaldau y Puimestre. Cuando se sembraban los tres primeros, los cuatro restantes permanecían en barbecho, formándose así dos añadas (DAumas, 1976: 252). 
demográfico en la segunda mitad del siglo XVI, relacionado con el aumento de la cabaña ganadera, la ausencia de enfermedades y pestes, y la buena climatología. Las normas concejiles trataron de poner freno a la puesta en cultivo de nuevos campos (Gómez de Valenzuela, 2003). El origen de los panares puede ponerse en relación con esta situación, pues su implantación permitió responder tanto a la necesidad de tierras de cultivo como al mantenimiento de los pastos comunales. La puesta en producción agrícola de estas tierras solo era posible gracias al estiércol generado por el ganado ${ }^{10}$.

Una segunda cuestión es si el espacio destinado a los panares ha permanecido estable a lo largo del tiempo. Por un lado, hay que considerar el factor climático. Los panares son zonas de cultivo que alcanzan o incluso superan el límite altitudinal tolerable para el cereal, pero la cantidad de horas de insolación anuales es aún más determinante que la altitud (Balcells \& Creus-Novau, 1986). Precisamente por ello se reservaban las zonas superiores de las laderas para el cereal, mientras los fondos de valle, mucho más húmedos y menos soleados, abundaban más en prados ${ }^{11}$. Este límite altitudinal, sin embargo, no ha sido el mismo a lo largo del tiempo. Conocemos cada vez mejor, a partir de los recientes estudios paleoclimáticos, las variaciones del clima a lo largo de la historia, incluso con precisión de décadas (Monserrat, 2009; González-Sampériz et al. 2017; Pérez-Sanz et al. 2011). Desde esta perspectiva, parece improbable que se cultivara cereal en la parte superior de los panares, que llegaba a más de $1800 \mathrm{~m} \mathrm{s.} \mathrm{n.} \mathrm{m.} \mathrm{durante} \mathrm{las} \mathrm{fases} \mathrm{más} \mathrm{críticas} \mathrm{de} \mathrm{la} \mathrm{Pequeña} \mathrm{Edad} \mathrm{de} \mathrm{Hielo} \mathrm{(1300-1850}$ d. C.). Sin embargo, esto no puede hacernos descartar que, en fases más cálidas, estas zonas altas fueran puestas en cultivo con anterioridad. En el periodo cálido medieval (9001300 d. C.), las ocupaciones y los aprovechamientos agrícolas pudieron llegar a altitudes superiores a las actuales (Ruas, Rendu \& Bergeret, 2005). Las evidencias son numerosas: el cultivo de la vid y del olivo llegaron también muy por encima de su límite actual (Azcárate, 1990).

También la línea que delimitaba lo que se consideraba panar, y que por tanto estaba sujeto a regulación, y lo que no, debió ser objeto de oscilaciones constantes y de negociación continua. Además del climático, el otro factor determinante fueron los cambios socioeconómicos relacionados con el ganado. El ovino convivía con el vacuno, en pro-

10. Es probable que algunas casas (no los grandes propietarios) tuvieran su ganado fundamentalmente como medio de abonar sus campos.

11. En cambio, en valles de la vertiente francesa del Pirineo central, como el de Campan, los cultivos ocupaban preferentemente el fondo del valle y la parte baja de las laderas, mientras los prados se extendían más arriba (CAVAILlĖs, 1931: 97-99). El fondo de estos valles es mucho más amplio y plano, como consecuencia de la acción de unos glaciares de mucho mayor desarrollo respecto de los de la vertiente española, lo que les asegura una buena insolación. Por esta razón, no necesitaron panares. 
porciones muy variables según los valles, y con el recrío de mulas, que abrió una nueva vía económica para los valles altos (Fillat, 1980b, 2003), por lo menos desde la segunda mitad del siglo XVIII ${ }^{12}$. Los montañeses compraban mulas jóvenes y yeguas en Francia, las recriaban con los pastos de los prados de siega durante dos años y posteriormente las vendían en las ferias de ganado. Esta dedicación fue especialmente importante en los valles de Hecho, Tena y Benasque (Ballarín, 1968: 76), se reactivó como consecuencia de la puesta en cultivo de las tierras desamortizadas y se extinguió como consecuencia de la mecanización del campo a mediados del siglo xx.

A pesar de las dificultades derivadas de la desamortización, con la venta a particulares de grandes extensiones de eriales y la fragmentación y el encarecimiento de los pastos de invernada, la trashumancia de ovino siguió vigente hasta mediados del siglo $\mathrm{xx}$ en la mayor parte de los valles. El hundimiento del sistema fue consecuencia de la escasez de pastores provocada por la emigración masiva a partir de los años cincuenta y sesenta y los avances en las técnicas frigoríficas para la conservación de la carne, que propiciaron la sustitución del ovino por el vacuno. Este cambio de orientación ganadera tuvo consecuencias en el espacio destinado a los panares. El vacuno permanecía en el valle en invierno, por lo que había que proveer su manutención. Por tanto, aumentaron los prados de siega en detrimento del cereal ${ }^{13}$, incluso en los panares. Por otra parte, habría que considerar también en esta evolución la importancia de la introducción del cultivo de la patata a mediados del siglo XVIII (Abad, 2016). Este cultivo está mucho más adaptado al terreno de alta montaña que el cereal y permitió una alternativa al barbecho con la introducción de rotaciones más complejas. Desconocemos el proceso de introducción, pero presumiblemente se implantó primero en los campos primarios alrededor del pueblo, en los que se practicaba un tipo de alternancia de cereal y patatas, a menudo dividiendo las parcelas cultivadas. Tras la recogida de la cosecha del cereal se sucedía un semibarbecho invernal, interrumpido el mes de mayo o junio por la siembra de patatas (Daumas, 1976: 324-325). Al extenderse este sistema a los panares, entró en conflicto con el barbecho obligatorio regulado por las añadas. Además, los rendimientos muy superiores de este cultivo respecto del cereal en estas condiciones climáticas permitieron reducir el espacio destinado a asegurar la alimentación de la población, y por tanto detraer espacio al cereal en beneficio del prado (Monserrat, 2014).

En la actualidad, los panares como tales han desaparecido por el abandono de los cultivos en altitud. Sin embargo, a pesar de su degradación como consecuencia del aban-

12. El comisario regio Francisco Zamora, en viaje por el Alto Aragón en 1794, anota 800 mulas de recrío en el valle de Benasque, 400 en Gistaín, 200 en Broto y 400 en el valle de Tena (BuIL, 1997). 13. El cambio se produce, según los valles, desde principios hasta a mediados del siglo XX. 
dono, que provoca la colonización espontánea de la vegetación en muchos casos, su impronta en el paisaje sigue muy presente en aquellos lugares en donde los campos se reconvirtieron en praderas de siega o en simples pastos a diente. El paisaje en forma de terrazas generado en los panares, con una sucesión ininterrumpida de fajas separadas por pequeños taludes herbosos, es uno de los más característicos y particulares que podemos encontrar en estas áreas de montaña, representativo de los usos tradicionales de la montaña y la adaptación humana al territorio con cultivos en altitud y pendiente.

\section{FIGURA 1}

\section{Panar de Viadós en Gistaín}

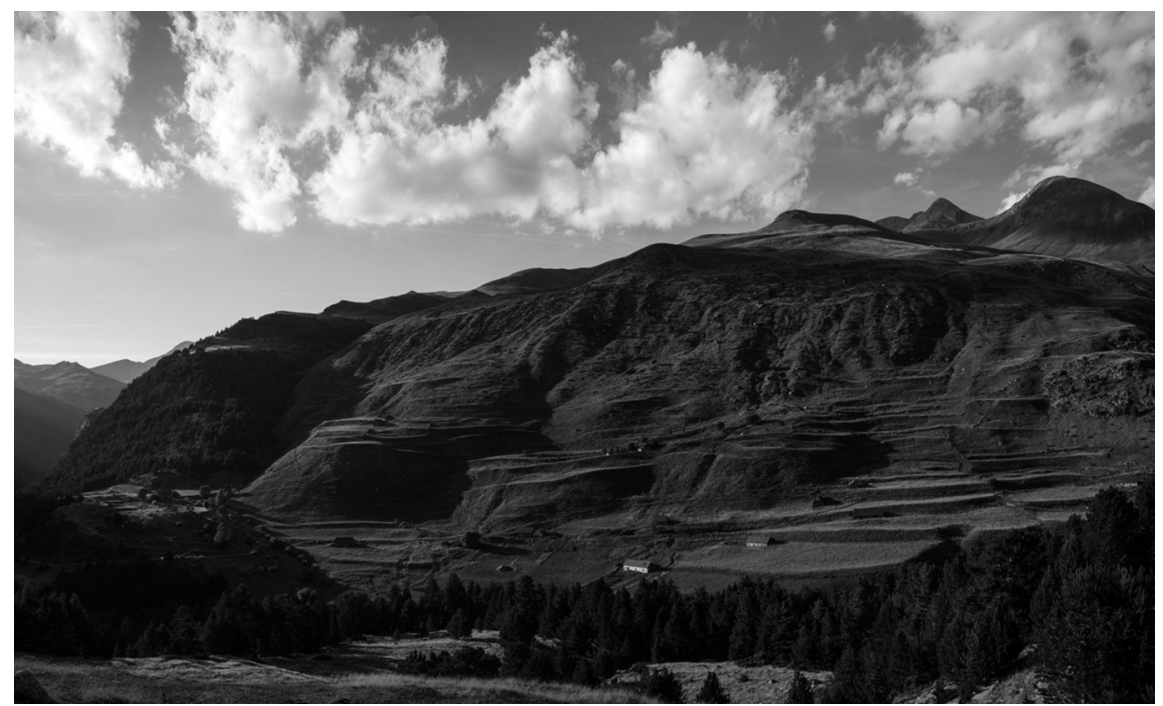

Fuente: fotografía de Carlos Fernández Piñar.

\section{DISTRIBUCIÓN DE LOS PANARES EN EL PIRINEO ARAGONÉS}

Según Daumas, antiguamente los panares se extendían por toda la cadena pirenaica, pero desaparecieron mucho antes en la vertiente francesa (Cavailllès, 1931; Chevalier, 1956; Daumas, 1961: 17) ${ }^{14}$. En la parte española perduraron hasta los años sesenta del pasado siglo desde Navarra ${ }^{15}$ hasta Cataluña, con especial incidencia en el tramo aragonés

14. Cavaillès cita el Règlement de Laruns (valle de Ossau), de 1587, en el que se habla de cultivos situados dentro del espacio comunal, vedados al paso de los ganados (CAVAILLÈs, 1931: 102). En el valle de Bastan, en el alto valle de Héas, cita también cultivos de cereales asociados a prados de siega (Ibid.: 224), pero desconocemos si tenían las características enunciadas de los panares.

15. Daumas (1961: 17) da la referencia de panares en el área navarra por información oral del profe- 
desde el valle de Tena al de Barrabés (Daumas, 1976:252; 1961: 17). No parece que existieran en los valles occidentales, donde predominaron los cultivos en pendiente e incluso procedimientos de agricultura nómada (articas). Esta diferencia puede estar relacionada con aspectos climáticos o con una organización pastoril -bien detallada por Pallaruelo (1993: 34)-, caracterizada por el uso de los aborrales (pastos situados en las sierras prepirenaicas, a medio camino entre las poblaciones de origen y los pastos de invernada), que eran inexistentes en los valles orientales (Garcia Ruiz, 1976). Mientras, en los valles orientales, se desarrollaron complejos sistemas de abancalamiento y terrazas (García-Ruiz et al., 2015).

\section{FIGURA 2}

\section{Distribución de panares en el Alto Aragón}

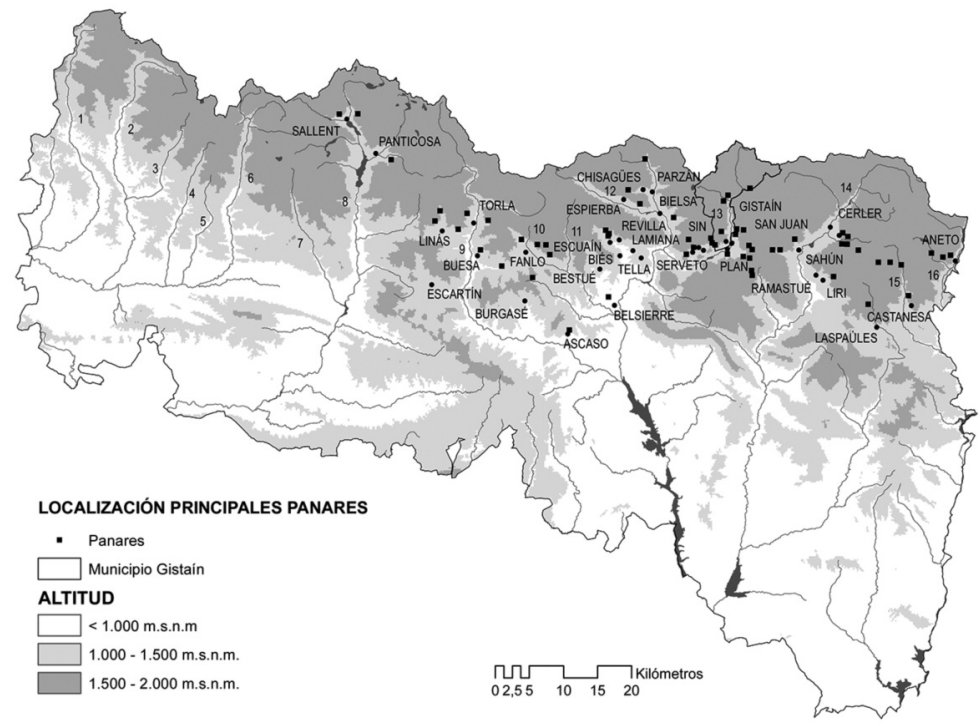

(1) valle de Ansó; (2) valle de Hecho; (3) valle de Aragüés; (4) valle de Aísa; (5) valle de Borau; (6) valle de Canfranc; (7) valle de Acumuer; (8) valle de Tena; (9) valle de Broto; (10) valle de Vió; (11) valle de Puértolas; (12) valle de Bielsa; (13) valle de Gistaín; (14) valle de Benasque; (15) valle de Castanesa; (16) valle de Barrabés.

Fuente: elaboración propia.

Tampoco existieron panares en los valles de fondo plano amplio, de origen glaciar. Este es el caso de Benasque, o el de muchos valles franceses, como los de Aure o Campan. En ellos los cultivos encontraron espacio suficiente y buena insolación sin tener que recurrir

sor Alfredo Floristán Imízcoz, de la Universidad de Navarra. Posiblemente se trate de los panificados. 
a las laderas. Era en los valles estrechos donde la escasez de suelo llano obligó a llevar los cultivos a las zonas altas más favorables y a ponerlos en contacto con los pastos comunales. A partir de estas referencias, de la toponimia y del estudio de las ortofotos proponemos una relación, con carácter provisional, de los panares que se distribuyen por la geografía del Alto Aragón (Figura 2 y Cuadro 1).

\section{CUADRO 1}

Relación provisional de panares en el Alto Aragón

\begin{tabular}{|c|c|c|c|}
\hline PANARES & LOCALIDAD & MUNICIPIO & COMARCA \\
\hline Travenosa & Panticosa & PANTICOSA & ALTO GÁLLEGO \\
\hline Tresarrato de Pondiellos & Sallent de Gállego & SALLENT DE GÁLLEGO & ALTO GÁLLEGO \\
\hline Diera, Angarrués, El Casar & Espierba & BIELSA & SOBRARBE \\
\hline Costadiera & Las Cortes & BIELSA & SOBRARBE \\
\hline Angarrués y Sopacas & Chisagüés & BIELSA & SOBRARBE \\
\hline Escagüés, Covilat & Bielsa & BIELSA & SOBRARBE \\
\hline Hospital de Parzán, Entrecruzes & Parzán & BIELSA & SOBRARBE \\
\hline Sesil, San Esteban & Buesa & BROTO & SOBRARBE \\
\hline La Solana, 0 Lomar & Fanlo & FANLO & SOBRARBE \\
\hline San Ginés & Ceresuela & FANLO & SOBRARBE \\
\hline La Poma, Viciele, Igüerra, Lisiert y Viadós & Gistaín & GISTAÍN & SOBRARBE \\
\hline Los Fenales & Nocito & NUENO & SOBRARBE \\
\hline Feneplán, Los Desvíos, El Chordal & Serveto & PLAN & SOBRARBE \\
\hline Arbas, Pitiligué, Sarramions, El Chordonal, Cimalitera, Lisé & Plan & PLAN & SOBRARBE \\
\hline $\begin{array}{l}\text { Habanera, Cundi Plana, San Miguel, Pui Tella, El Panaret, } \\
\text { Las Planas, Os Petrosos, } 0 \text { Billar, Las Balles }\end{array}$ & Bestué & PUÉRTOLAS & SOBRARBE \\
\hline $\begin{array}{l}\text { Estibeta, Yerri, Fontané, Estigües, Las Planas, Escrabés, } \\
\text { Ingrota, Fené Mayor, La Calva }\end{array}$ & San Juan de Plan & SAN JUAN DE PLAN & SOBRARBE \\
\hline Sevillún, panar de Arriba, panar de Abajo, Chardal & $\operatorname{Sin}$ & TELLA-SIN & SOBRARBE \\
\hline Solana de la cuenca del Yaga & Tella & TELLA-SIN & SOBRARBE \\
\hline Secalás, El Estrecho y Fragón & Revilla & TELLA-SIN & SOBRARBE \\
\hline Diazas, Lusarre & Torla & TORLA & SOBRARBE \\
\hline El Soaso, Toronzué & Linás de Broto & TORLA & SOBRARBE \\
\hline Santa Creu & Betesa & ARÉN & RIBAGORZA \\
\hline $\begin{array}{l}\text { La Rueda, Paluenga, Guáriz, Ubiergo, Clot, Micaldau } \\
\text { y Puimestre }\end{array}$ & Cerler & BENASQUE & RIBAGORZA \\
\hline Bruji & Liri & CASTEJÓN DE SOS & RIBAGORZA \\
\hline La Ribereta y Montaña de las Casas & Las Paúles & LASPAULES & RIBAGORZA \\
\hline Lestuy y La Menada & Aneto & MONTANUY & RIBAGORZA \\
\hline Santa Olaria y Sarronal & Estet & MONTANUY & RIBAGORZA \\
\hline $\begin{array}{l}\text { Posinqueso, Llana de Obeja, Llanos de Obarra, Basibé, } \\
\text { Fonchanina }\end{array}$ & Castanesa & MONTANUY & RIBAGORZA \\
\hline Llisat, La Sila, Llabarins & Sahún & SAHÚN & RIBAGORZA \\
\hline
\end{tabular}

Fuente: elaboración propia. 


\section{LOS PANARES DEL MUNICIPIO DE GISTAÍN}

El valle de Gistaín o Chistau se ubica en la zona central de la cordillera pirenaica, en la comarca de Sobrarbe, enclavado entre las altas cumbres del Pirineo axial y de las sierras interiores, con los macizos de Posets, Bachimala, Punta Suelza y Culfreda entre las primeras y el macizo de Cotiella, también rozando los $3000 \mathrm{~m} \mathrm{s.} \mathrm{n.} \mathrm{m.} \mathrm{en} \mathrm{las} \mathrm{segundas.} \mathrm{El}$ territorio del valle se organiza administrativamente en los municipios de Gistaín, San Juan de Plan, Plan, y Tella-Sin, con gran parte de este último fuera del valle, ya en la ribera occidental del río Cinqueta. El municipio de Gistaín ocupa el sector noroccidental y es el único con frontera internacional.

En este valle el sistema de los panares se mantuvo vigente hasta hace pocas décadas, y pueden encontrarse aún habitantes que lo trabajaron. El espacio productivo agrícola del municipio de Gistaín se organizaba hasta mediados del siglo pasado en una serie de conjuntos sucesivamente más alejados del núcleo de población. De mayor a menor proximidad a las casas estos eran:

- Los huertos, situados junto a las casas y en pequeñas parcelas regadas junto a los barrancos más próximos.

- Los campos primarios, situados alrededor del núcleo de población, estaban destinados a la producción de cereales, patatas y legumbres. Históricamente debieron ocupar en primer lugar los espacios con menor pendiente justo por debajo del pueblo ${ }^{16}$, donde se encuentran las mejores parcelas, y después se extenderían sobre el caserío, en terreno más abrupto, y hacia los barrancos laterales. En la partida de Troixo, en el tramo inferior del barranco de la Poma, rico en agua y sedimentos, se ubicaron la mayor parte de las huertas.

- La Ribera es el nombre que recibe el fondo de valle del Cinqueta, cerrado y estrecho, donde aparecían campos de cultivo y prados de siega, que se extendían también por las partes más bajas de los barrancos subsidiarios. Los prados estaban ligados fundamentalmente al ganado mayor, que no realizaba trashumancia invernal, pero también a las pocas ovejas y cabras que quedaban en el pueblo en la estación invernal.

16. En la parte baja del municipio, lindando con Plan, se encuentra la partida de Chesta, que parece estar en el origen del nombre del valle, Chestave-Chestau-Chistau (BLAs \& Romanos, 2008: 188), lo que confirmaría que fueron los primeros campos en ocuparse. Las mejores parcelas pertenecen a las casas más ricas y antiguas. 
- Los panares, que constituían otro escalón en la distribución territorial de los aprovechamientos agrícolas, ocupaban las terrazas de obturación glaciar sobre el fondo de valle.

- Por último, algunas parcelas ganadas al monte en zonas de difícil acceso se cultivaron de forma intermitente solo en periodos de necesidad. Por ejemplo, en los tiempos de máxima presión demográfica a finales del siglo xIX y en el primer tercio del siglo $\mathrm{xx}^{17}$.

En total, la superficie agrícola útil no sobrepasaba las seiscientas hectáreas, contando los prados de siega, y representaba tan solo el $8 \%$ del total del municipio. Las parcelas eran pequeñas y su propiedad estaba enormemente dispersa, de forma que cada propietario disponía de pequeños campos en partidas muy distantes entre sí. Este sistema tenía una clarísima desventaja por la cantidad de desplazamientos que se debían realizar, pero gracias a la dispersión geográfica la producción era menos vulnerable a fenómenos atmosféricos muy localizados, como las tormentas, porque alguna zona siempre podía salvarse. La diversificación, tanto en los tipos de cultivo como en su disposición en el espacio, era una de las principales estrategias del campesino ante la incertidumbre de unas cosechas que dependían de unas condiciones climáticas muy difíciles para la agricultura.

En Gistaín existieron cinco panares (Igüerra, La Poma,Viciele, Lisiert y Viadós), que se organizaban en dos añadas. Igüerra, Viciele y Lisiert, formaban la añada de Viciele. La Poma y Viadós, el más alejado de la población, constituían la añada de La Poma (Figura 3). Para la interpretación de estas áreas, se ha recurrido a tres fuentes principales: el actual catastro para la distribución en parcelas, el primer amillaramiento de 1863 para conocer el uso de cada parcela a mediados del siglo xIx, y la información oral recogida mediante entrevistas con los habitantes de más edad de Gistaín para poder relacionar los anteriores datos.

La distribución parcelaria actual no ha sufrido grandes variaciones con respecto a la existente en los siglos XIX y Xx. Los cambios más importantes se refieren más a la propiedad de las parcelas que a su número y tamaño. El sistema de heredero único propio de las montañas (Comas \& Pujadas, 1994) evitó la disgregación del patrimonio, mientras la dispersión en la distribución espacial de la propiedad y la ausencia de muchos propietarios emigrados impidieron la concentración parcelaria. Pudo producirse un pequeño

17. No se puede asegurar que se trate de articas, pues, aunque actualmente están abandonadas y no parece que se cultivaran con continuidad durante muchos años, no se trataba de cultivos itinerantes. Algunas contaron con cabaña e incluso con borda. 
incremento del número de parcelas puestas en cultivo entre finales del siglo XIX y principios del xx como consecuencia del aumento de la población ${ }^{18}$, pero podemos considerar la parcelación actual, al menos en su inmensa mayoría, como la misma que habría a mediados del siglo $\mathrm{XIX}^{19}$.

Esta parcelación se ha contrastado con la registrada en el primer amillaramiento de $1863^{20}$. Esta declaración no nos proporciona una documentación cartográfica. El primer documento catastral disponible que cuenta con una representación gráfica de las parcelas y de su ubicación en el territorio está dibujado sobre los fotogramas del vuelo 195657 serie B, llamado vuelo americano. Esto supone una gran desventaja para estudiar la distribución de los cultivos en relación a lo que sucede en la vertiente francesa de la cordillera, donde se dispone de planos catastrales completos desde el primer tercio del siglo XIX.

El amillaramiento nos aporta una relación de las parcelas por propietario, la partida en que se encuentran, el aprovechamiento (prácticamente sólo distingue cereal o prado y, en muy contadas ocasiones, hortalizas, aparte de los huertos), su calidad (primera, segunda o tercera) y su superficie, medida en fanegas y almudes ${ }^{21}$. A partir de esta información, y con ayuda de los informantes locales para ubicar las partidas referidas, se han identificado las que formaron cada uno de los panares. La casa a que corresponde el nombre de cada propietario se ha establecido a partir de la consulta de datos en el Ayuntamiento de Gistaín, donde se conserva copia del amillaramiento con anotaciones que permiten establecer la relación.

El carácter fiscal de los amillaramientos hace que podamos tener dudas respecto de la fiabilidad de sus datos. Algunos investigadores han opinado que en la época de su implantación los campesinos de este ámbito geográfico no escondían parcelas, pero disminuían la superficie de cada una de ellas, con objeto de rebajar el impuesto a pagar. Estos autores calcularon que de media la disminución de la superficie real era de un $30 \%$

18. En zonas de accesos muy difíciles y condiciones de cultivo precarias, como la partida de La Fesa o El Chelau, en las que aún se reconocen campos de labor en parcelas que no llegaron ni siquiera a ser registradas en el catastro.

19. Las parcelas registradas en el amillaramiento de 1863 y en el catastro actual presentan un alto nivel de concordancia, en un trabajo de cotejo aún en proceso. En el Cuadro 3 se puede ver el caso de las propiedades de varias casas.

20.Archivo Histórico Provincial de Huesca (AHPHU), H-000708. Al tiempo de la edición de este trabajo ha aparecido en el Archivo Municipal de Gistaín otro amillaramiento anterior, fechado en el año 1854, del que no hay constancia en el AHPHU.

21.La conversión a hectáreas es posible con una equivalencia de 1 fanega $=12$ almudes $=0,0715$ ha, y 1 almud $=60 \mathrm{~m}^{2}$. 
CUADRO 2

Partidas incluidas en los panares, extraídas del amillaramiento de 1863

\begin{tabular}{|c|c|c|c|c|c|c|c|c|c|c|c|c|c|}
\hline 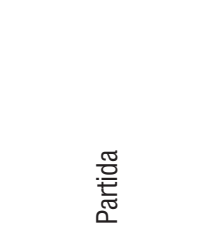 & 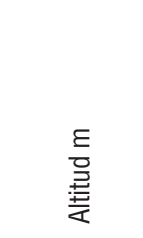 & 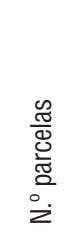 & 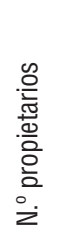 & 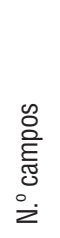 & $\begin{array}{l}\mathscr{0} \\
\frac{0}{\pi} \\
\frac{\pi}{0} \\
\dot{z}\end{array}$ & 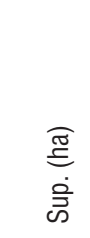 & 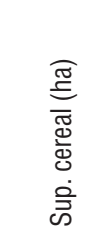 & 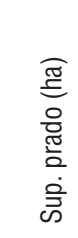 & 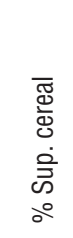 & 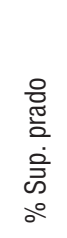 & 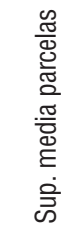 & 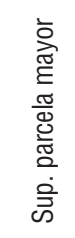 & \\
\hline \multicolumn{14}{|l|}{ Panar de Igüerra } \\
\hline Tozal de Igüerra & $1700-1775$ & 7 & 7 & 7 & & 1,03 & 1,03 & & 100 & 0 & 0,15 & 0,29 & \\
\hline Sobrelapila & $1650-1725$ & 7 & 7 & 7 & & 0,85 & 0,85 & & 100 & 0 & 0,12 & 0,17 &, 0 \\
\hline Cabo de Igüerra & $1625-1700$ & 14 & 13 & 14 & & 4,96 & 4,96 & & 100 & 0 & 0,35 & 1,03 & \\
\hline La Cruz de Igüerra & $1580-1625$ & 4 & 4 & 4 & & 1,12 & 1,12 & & 100 & 0 & 0,28 & 0,57 & \\
\hline El Sarrués & $1525-1725$ & 7 & 7 & 7 & & 1,94 & 1,94 & & 100 & 0 & 0,25 & 0,63 & 0,0 \\
\hline TOTAL & & 39 & & 39 & 0 & 9,89 & 9,89 & 0,00 & 100 & 0 & 0,25 & 1,03 & 0,04 \\
\hline \multicolumn{14}{|l|}{ Panar de La Poma } \\
\hline El Chelau & $1800-1900$ & 5 & 5 & 5 & & 1,60 & 1,60 & & 100 & 0 & 0,32 & 0,79 & \\
\hline Belicuengas & $1800-1850$ & 6 & 6 & 5 & & 1,86 & 1,72 & & 92 & 0 & 0,31 & 0,57 & 1 \\
\hline Las Fuens & $1800-1825$ & 1 & 1 & 1 & & 0,32 & 0,32 & & 100 & 0 & 0,32 & 0,32 & ,3 \\
\hline Cabo la Poma & $1775-1850$ & 35 & 30 & 35 & & 9,22 & 9,22 & & 100 & 0 & 0,26 & 0,72 & 0,0 \\
\hline La Ruyén & $1725-1800$ & 3 & 3 & 3 & & 0,72 & 0,72 & & 100 & 0 & 0,24 & 0,32 & \\
\hline El Curtil & $1700-1725$ & 10 & 8 & 8 & 2 & 2,72 & 2,23 & 0,43 & 82 & 16 & 0,27 & 0,89 & \\
\hline Soplán & $1625-1750$ & 19 & 17 & 18 & 1 & 4,76 & 4,61 & 0,14 & 97 & 3 & 0,25 & 0,86 & 0,0 \\
\hline Chiradiello & $1575-1700$ & 22 & 19 & 19 & 5 & 5,44 & 4,44 & 0,48 & 82 & 9 & 0,25 & 0,73 & 0,0 \\
\hline TOTAL & & 101 & & 94 & 8 & 26,64 & 24,86 & 1,05 & 93 & 4 & 0,27 & 0,89 & \\
\hline \multicolumn{14}{|l|}{ Panar de Viciele } \\
\hline Cabo Biciele & $1700-1825$ & 5 & 5 & 5 & & 1,59 & 1,59 & & 100 & 0 & 0,34 & 0,89 & \\
\hline La Coda & $1700-1825$ & 7 & 7 & 7 & 1 & 1,20 & 1,06 & 0,14 & 88 & 12 & 0,17 & 0,21 & \\
\hline Sullert & $1625-1700$ & 2 & 2 & 2 & 1 & 0,49 & 0,37 & 0,12 & 76 & 24 & 0,29 & 0,29 & \\
\hline Viciele & $1525-1700$ & 52 & 38 & 45 & 8 & 11,65 & 10,07 & 1,58 & 86 & 14 & 0,22 & 0,64 & \\
\hline La Cernil & $1500-1700$ & 9 & 9 & 9 & & 2,52 & 2,52 & & 100 & 0 & 0,28 & 1,00 & \\
\hline TOTAL & & 75 & & 68 & 10 & 17,44 & 15,60 & 2,89 & 89 & 17 & 0,25 & 1,00 & \\
\hline \multicolumn{14}{|l|}{ Panar de Lisiert } \\
\hline Tozal de Lisiert & $1700-1775$ & 2 & 2 & 2 & & 1,01 & 1,01 & & 100 & & 0,51 & 0,38 & \\
\hline Lisiert & $1575-1700$ & 42 & 33 & 35 & 18 & 12,27 & 7,38 & 4,89 & 60 & 40 & 0,27 & 0,64 & \\
\hline TOTAL & & 44 & & 37 & 18 & 13,28 & 8,39 & 4,89 & 63 & 37 & 0,28 & 0,64 & \\
\hline \multicolumn{14}{|l|}{ Panar de Viadós } \\
\hline La Ballariza & $1800-1875$ & 4 & 4 & 2 & 1 & 0,74 & 0,45 & 0,29 & 61 & 39 & 0,18 & 0,31 & \\
\hline La Ribereta & $1690-1710$ & 1 & 1 & & 1 & 0,14 & & 0,14 & & 100 & 0,14 & 0,14 & \\
\hline Viadós & $1675-1850$ & 56 & 43 & 46 & 16 & 11,69 & 8,42 & 3,27 & 72 & 28 & 0,22 & 0,72 & \\
\hline Santa Olaria & $1675-1750$ & 4 & 4 & 4 & & 0,88 & 0,88 & & 100 & & 0,22 & 0,36 & \\
\hline Forcallo & $1625-1675$ & 3 & 3 & 3 & & 0,39 & 0,39 & & 100 & & 0,13 & 0,16 & \\
\hline TOTAL & & 68 & & 55 & 18 & 13,84 & 10,14 & 3,70 & 73 & 27 & 0,21 & 0,72 & \\
\hline
\end{tabular}

Nota: se reflejan las partidas incluidas en cada panar; su altitud; el número de parcelas y el número de propietarios; la superficie (convertida de fanegas y almudes a hectáreas) en cada una de ellas; el número de parcelas que contienen cultivo de cereal y prados; su superficie y el porcentaje del cultivo sobre el total de la partida; superficie media de las parcelas y superficie de la parcela más grande y más pequeña por cada partida.

Fuente: elaboración propia a partir de H-000708 (AHPHU). 


\section{FIGURA 3}

\section{Distribución de panares en el municipio de Gistaín}

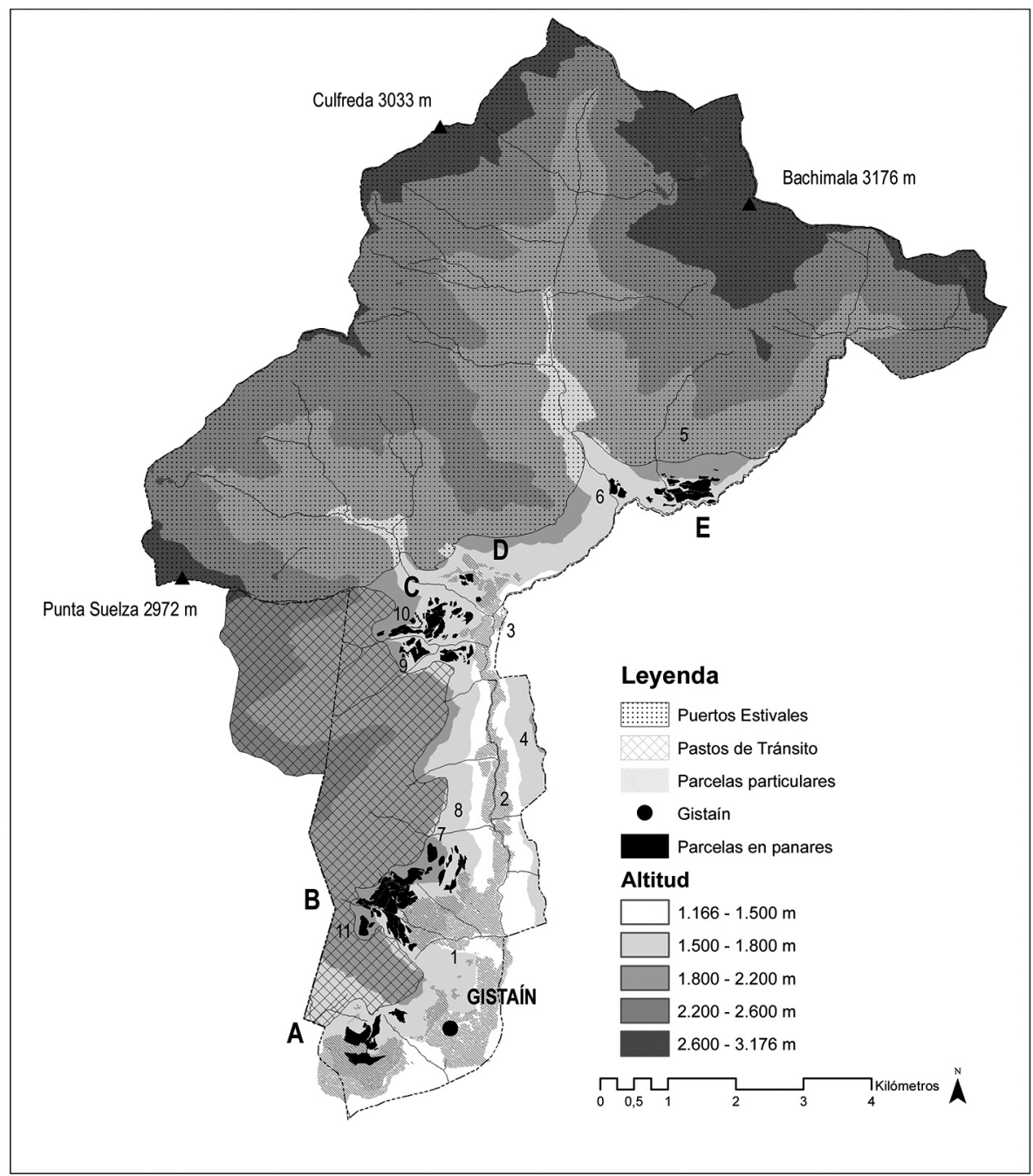

(A) panar de Igüerra; (B) panar de La Poma; (C) panar de Viciele; (D) panar de Lisiert; (E) panar de Viadós.

(1) ElTroixo; (2) La Ribera (fondo de valle del río Cinqueta); (3) Las Fontaciellas; (4) Las Feixas; (5) Orieles; (6) El Forcallo; (7) El Chelau; (8) La Fesa; (9) La Coda; (10) Cabo Viciele; (11) Las Fuens.

Fuente: elaboración propia.

(Cuesta, 2001; Daumas, 1976). La diferencia entre la superficie de las parcelas de los panares declarada y la real, que se ha comprobado en el trascurso de este trabajo, no es ni mucho menos constante. En el Cuadro 4 pueden verse algunos ejemplos de correlación 
entre la superficie declarada y la real de las parcelas actuales ${ }^{22}$. En cualquier caso, para el interés de este trabajo, más que la superficie exacta de las parcelas interesa su aprovechamiento y ubicación, por lo que una variación en la estimación de la superficie no supone grandes cambios en las conclusiones que puedan sacarse. A partir de estos datos, se han seleccionado las partidas correspondientes a cada uno de los panares (expresadas en el Cuadro 2).

El Cuadro 3 expresa el número de parcelas del catastro de mediados del siglo xx incluidas dentro de cada uno de los panares, según los límites marcados por la tradición oral. En total representan aproximadamente una cuarta parte de las parcelas y de la superficie particular del municipio. En la Figura 3 se señala su ubicación sobre el parcelario del catastro actual.

\section{CUADRO 3}

Parcelas del actual catastro incluidas en los panares a mediados del siglo $\mathrm{xx}$

\begin{tabular}{lcrrrrrrc}
\hline & Igüerra & La Poma & Viciele & Lisiert & Viadós & Total panares & Total municipio & \% panares sobre total \\
\hline N. ${ }^{\circ}$ Parcelas & 46 & 132 & 97 & 9 & 63 & 347 & 1308 & 26,53 \\
Superficie (ha) & 21 & 55,77 & 33,98 & 2,59 & 23,33 & 136,67 & 539,72 & 25,32 \\
\hline
\end{tabular}

Fuente: elaboración propia a partir del catastro.

\section{EL PAISAJE HUMANIZADO CHISTABINO}

Los dos componentes fundamentales del paisaje humanizado del municipio de Gistaín fueron -y siguen siendo- las praderas de siega, con sus bordas asociadas, y los panares en las laderas altas, donde casi todas las parcelas cuentan con $c_{a b a n a^{23}}$.

Las partes bajas de los valles subsidiarios, justo por debajo de los panares, presentan una composición paisajística compleja, formada por multitud de pequeñas parcelas en las que abundan los prados, separadas por un reticulado de seto vivo, árboles y arbustos en-

22. Independientemente de la existencia o no de una voluntad de ocultación, los sistemas metrológicos anteriores a la implantación del metro y sus derivados, ni eran tan precisos, ni existía la necesidad social de que lo fueran (KULA, 1980). La disparidad entre los datos convertidos provenientes del amillaramiento y los del actual catastro, que no sigue un patrón porcentual claro, puede deberse al concepto de las medidas tradicionales, basadas en la cantidad de trabajo necesario para poner en producción la tierra o en la cantidad de semilla necesaria para su puesta en cultivo, y no en sus dimensiones físicas.

23. Las praderas de siega siempre existieron, a juzgar por la documentación antigua, aunque en menor medida que en la actualidad. Los panares, aunque perdido su uso, siguen constituyendo parte del paisaje. 
tre los que destacan los fresnos, que cumplían además una función forrajera, pues sus ramas y hojas se recolectaban en otoño como complemento alimenticio invernal de los ganados (Fillat et al., 1984). Se trata del tipo de paisaje que se define por el término francés bocage, sin traducción en castellano, claro ejemplo de paisaje humanizado, pues no se trata de una composición natural, sino resultado de una prolongada actividad humana. $\mathrm{Su}$ origen debe estar precisamente en el deseo de segregar estas zonas de prados de las recorridas por los rebaños comunales. Hasta mediados del siglo XIX sólo los prados de mayor valor, que podían ser regados y por tanto ofrecían varios cortes, se cerraban con setos (Daumas, 1976: 133-135; Biarge, 2009: 59). Con el posterior abandono de muchos campos de cereal y su sustitución por prados, este procedimiento se extendió. El seto cumplía no solo una función delimitadora y protectora, sino que además aportaba humedad, estabilidad y abono con la hoja muerta, de modo que su papel era fundamental en el sistema de praderías (Monserrat, 1992: 66-67; Monserrat \& Villar, 1995: 165; Montserrat, 2009:126). En estos espacios la construcción característica era la borda, que permitía en planta baja la estabulación invernal del ganado, fundamentalmente del vacuno, y el acopio de la hierba para su alimentación en el pajar situado en el piso superior.

Más arriba, a partir de la cota 1500-1600 m de altitud, los prados prácticamente desaparecían y el centeno ocupaba la casi totalidad de las parcelas, lo que generaba un paisaje diferente, de suaves terrazas conectadas entre sí por taludes herbosos formando laderas abancaladas. La hierba que crecía en los márgenes del cultivo de cereal (espuenas, espuendas o marguins) también era objeto de aprovechamiento, se segaba y se mezclaba con la paja del centeno, que se almacenaba en las bordas como forraje invernal ${ }^{24}$. Estas zonas altas constituyeron los panares, en los que no existían delimitaciones físicas de las parcelas dedicadas al cereal, que al ser cosechadas quedaban de pasto libre para los ganados. Allí no había casi ninguna borda, y la construcción más habitual eran las $c a-$ banas, construidas en casi todas las fincas para guardar las baranas y otros útiles y cobijar al pastor.

\section{RÉGIMEN DE USO DE LOS PANARES DE GISTAÍN}

El funcionamiento de los panares estaba regulado por la costumbre y el común acuerdo entre los vecinos. El régimen hacia el primer tercio del siglo xx lo conocemos a través de la tradición oral y de un trabajo inédito de Joaquín Villa (1986), natural de Gistaín, que recogió el sistema a mediados de la década de 1980 tras consultar a los abuelos que lo

24. Las espuenas, dependiendo del valle, pueden pertenecer al común, a la parcela por encima, por debajo, o compartirse entre las parcelas limítrofes (DAUMAS, 1976: 124). 
usaron en el periodo anterior a la Guerra Civil $^{25}$. La secuencia temporal sigue el esquema general para el conjunto del Pirineo oscense expuesto en el apartado 2 .

A partir del día de la Santa Cruz de Mayo (3 de mayo), se vedaban los panares a fin de preservar el pasto para la llegada de los rebaños trashumantes ${ }^{26}$, de modo que se prohibía la entrada de animales no solo en el comunal, sino también en las fincas particulares. Solo se admitía la entrada en fincas propias de dos bestias de carga y de hasta tres ovejas o cabras de leche, que en el caso de las familias más pobres suponían un último recurso alimenticio. La prohibición se mantenía hasta que todos los propietarios hubieran acabado las operaciones agrícolas de siega y mallado del cereal ${ }^{27}$ (Villa, 1986). El ganado que había permanecido en el pueblo sólo podía pastar en la femalla, nombre que se daba a los pastos de tránsito por encima de la cota de los panares, mientras las ovejas casazas $^{28}$, divididas en dos rebaños, se conducían a las partidas de Fontaciellas y Las Fei$\mathrm{xas}^{29}$.

A finales del mes de mayo llegaban al valle los rebaños trashumantes, recrecidos con las crías nacidas en la estancia en tierra baja. A su llegada, iban directos a los pastos del panar previamente reservado para ellos, sin pasar por el pueblo. Naturalmente se trataba de los panares de la añada en barbecho, pues la contraria, cultivada, permanecía vedada desde su siembra el año anterior hasta la recogida de la cosecha. A partir del día siguiente cada ganadero separaba sus animales, formando rebaños particulares. Por espacio de casi dos meses, hasta el 25 de julio, el llamado mes de la cabana, las ovejas permanecían en estos pastos, abonando las parcelas. Para ello, se encerraba el ganado en el barandau, que se cambiaba de posición cada día, de forma que el abonado se hiciera uniformemente. A esto se llamaba fer barana.

25. La obra de Joaquín Villa (1986), dirigida por D. Gaspar Mairal, profesor de Antropología Social de la Facultad de Ciencias Empresariales de la Universidad de Zaragoza y presentada al Premio Nacional de Investigación sobre Artes y Tradiciones Populares "Marqués de Lozoya", con el seudónimo Fabirol, obtuvo mención honorífica, con fecha de 19 de marzo de 1988.

26. Estos rebaños se organizaban en una serie de compañías, cada una de las cuales reunía los animales de varios propietarios que se asociaban para el arrendamiento de los pastos de invernada y para costear los pastores y los gastos de la trashumancia.

27. En el valle de Gistaín no existían las eras, de modo que la operación de la trilla se realizaba por majado del cereal en un terreno aplanado y acondicionado para ello en los mismos campos o junto a una borda. Esto además permitía conservar la larga paja del cereal entera, que tenía múltiples usos: como forraje invernal, mezclada con la hierba de las praderas de siega; como material de cubrición para las bordas y, antiguamente, incluso para las viviendas; y como materia prima para la elaboración de múltiples aperos agrícolas.

28. Nombre que recibían las ovejas que permanecían en el pueblo en invierno, sin trashumar.

29. Se trata de partidas de pastos ganados al bosque en terreno muy escarpado en la ladera izquierda del río Cinqueta (Figura 3). 
Para subir a los puertos estivales, se formaban dos compañías, entre las que se repartían todas las casas del pueblo. Cada una de ellas se dividía a su vez en dos rebaños, vigilado por sendos pastores, que repartían de forma proporcionada los animales de cada casa entre los dos. Además, se formaban otros dos rebaños, uno con todas las corderas y otro con todos los borregos, que subían antes a la montaña, hacia el 15 de junio (Villa, $1986)^{30}$. Los campos se araban inmediatamente, en cuanto el ganado subía a puerto, para que el abono quedase bien incorporado a la tierra, sin esperar a que lo consumiera el agua o el sol. A los quince días se volvía a dar otra vuelta a la tierra para evitar que empezara a salir hierba y se comenzaba a sembrar a partir de mediados de agosto. Hasta el final de este mes se sembraba el centeno y en septiembre, el trigo, para cosechar a finales de agosto o en septiembre del año siguiente, con el ciclo largo anteriormente referido.

Después de la estancia en los puertos, los rebaños comunales formados para el periodo estival bajaban hacia los pastos de tránsito, para aprovechar los rastrojos de los panares de la añada recién cosechada, a la que se había levantado ya la veda. Al ser estos rebaños comunales mucho más numerosos que los particulares del periodo primaveral, el abonado de los campos no se hacía entonces encerrando el ganado en corrales móviles, sino que el rebaño dormía suelto vigilado por el pastor; además, el propietario del campo que se iba a abonar cada noche estaba obligado a subir a guardar el ganado y llevar la cena al pastor. A partir del 1 de noviembre, festividad de Todos los Santos, se disolvían los rebaños comunales y cada propietario quedaba de nuevo al cuidado de sus animales, que permanecían en los panares y el común inmediato durante todo el mes de noviembre hasta el momento de trashumar al valle del Ebro.

Durante el periodo invernal, el centeno de los panares servía también como pasto a las ovejas casazas, excepto a las preñadas, pues había riesgo de que malpariesen. Los primeros brotes eran consumidos por este ganado antes de Navidad y de nuevo en marzo. Esto no acababa con el sembrado, sino al contrario, permitía que la raíz se conservara bajo la nieve sin pudrir y brotara mejor al llegar la primavera (Villa, 1986).

De todos modos, podemos observar diferencias importantes en la forma de uso de los distintos panares. Los más importantes eran La Poma y Viciele, por lo que daban nombre a cada una de las añadas. Junto con Igüerra, que se incluía en la añada de Viciele para compensar que La Poma tenía más parcelas, son los panares que se encuentran en contacto con la femalla por su parte superior, mientras que en las cotas inferiores enlazan con

30. Villa se refiere al primer tercio del siglo xx. El sistema sin duda fue variable en el tiempo, dependiendo del número de cabezas. En el siglo XIX se formaban cuatro compañías, según documento consultado en el archivo municipal, mientras que después de la guerra solo era una. 
el bocage del fondo de valle. Los tres eran utilizados como pastos en el periodo primaveral por el ganado ovino. En cambio, Lisiert y Viadós están ya en continuidad física con los pastos estivales, que estaban vedados hasta la apertura de los puertos el día de Santiago. Estos panares, en el periodo primaveral, recibían ganado vacuno, que pernoctaba en las numerosas bordas que allí se encuentran. Esto explicaría que a mediados del siglo $\mathrm{xx}$ muy pocas parcelas de Lisiert siguieran teniendo la consideración de panar, mientras que en los datos de 1863 ya observamos una mayor proporción de prados respecto del resto de los panares, aun cuando la mayor parte de las parcelas se dedicaban al cereal (véanse Cuadros 2 y 3). Por su parte, el panar de Viadós se reservaba para el ganado de labor, pero al seguir la alternancia de añadas sólo se empleaba para tal fin un año de cada dos. Tanto en Lisiert como en Viadós el abonado de los campos se hacía principalmente con el estiércol acumulado en los establos de las bordas. Solamente a la bajada de los puertos, y no en primavera, estercolaba el ganado ovino.

El régimen alternativo de cultivo entre los panares llevaba a los campesinos a tratar de disponer de parcelas más o menos equivalentes en cantidad y superficie en las dos añadas, de forma que tuvieran posibilidades similares de cosecha todos los años. Esto no siempre era posible, dentro de la heterogeneidad de los patrimonios de las distintas casas. Las más pudientes disponían de un conjunto de parcelas abundante y bien distribuido en todos los sectores, mientras otras tenían superficies muy descompensadas entre una y otra añada, o incluso sólo tenían cultivos en una de ellas ${ }^{31}$. En el Cuadro 4 se aportan algunos ejemplos a partir de los datos obtenidos del amillaramiento de 1863:

31. Entre las casas más pobres se daban todo tipo de situaciones. Casos como los de Casa Grima, cuyas tres propiedades estaban situadas en panares, o el de Casa Ciella, con ninguna de sus cuatro fincas en panares. 


\section{CUADRO 4}

Relación de propiedades en los panares de Casa Saila, Casa Micalé, Casa Cabo y Casa Cañau en el amillaramiento de 1863

\begin{tabular}{|c|c|c|c|c|c|c|c|c|c|c|}
\hline & \multirow[t]{2}{*}{ Panar } & \multirow[t]{2}{*}{ Partida } & \multirow[t]{2}{*}{ Cultivo } & \multicolumn{2}{|c|}{ Superficie } & \multirow[t]{2}{*}{ Calidad } & \multicolumn{2}{|c|}{$\begin{array}{r}\text { Superficie (ha) } \\
\text { actual }\end{array}$} & \multirow[t]{2}{*}{$\begin{array}{l}\text { Parc. } \\
\text { real }\end{array}$} & \multirow[t]{2}{*}{ Sup. } \\
\hline & & & & Fan. & Alm. & & ha & ha sum & & \\
\hline \multicolumn{11}{|c|}{ Casa Saila: 15 parcelas en propiedad, 7 en panares } \\
\hline \multicolumn{11}{|l|}{ Añada La Poma } \\
\hline & La Poma & Cabo la Poma & Cereal & 7 & & $3 .^{a}$ & 0,5005 & 0,5005 & 230 & 0,5083 \\
\hline & La Poma & Las Fuens & Cereal & 4 & 6 & $3 .^{a}$ & 0,322 & 0,322 & 137 & 0,4566 \\
\hline & Viadós & Viadós & Prado & 6 & 7 & $3 .^{a}$ & 0,471 & 0,471 & 24 & 0,5306 \\
\hline & Viadós & Viadós & Cereal & 4 & & & 0,286 & 0,286 & 42 & 0,2553 \\
\hline & & & & 21 & 13 & & 1,5795 & 1,5795 & & 1,7508 \\
\hline \multicolumn{11}{|l|}{ Añada Viciele } \\
\hline & Igüerra & Igüerra & Cereal & 4 & & $2 .^{a}$ & 0,286 & 0,751 & 234 & 1,6019 \\
\hline & & & Cereal & 6 & 6 & $3 .^{a}$ & 0,465 & & & \\
\hline & Viciele & Viciele & Cereal & 4 & & $3 .^{a}$ & 0,286 & 0,286 & 122 & 0,5179 \\
\hline & Viciele & El Estaso & Prado & 2 & & $3 .^{a}$ & 0,143 & 0,14311 & $1+112$ & 0,0934 \\
\hline & & & & 16 & 6 & & 2,7595 & 2,7595 & & 2,2132 \\
\hline \multicolumn{11}{|c|}{ Casa Micalé: 25 parcelas en propiedad, 8 en panares } \\
\hline \multicolumn{11}{|c|}{ Añada La Poma } \\
\hline & La Poma & Cabo la Poma & Cereal & 8 & & $3 .^{a}$ & 0,572 & 0,572 & 233 & 0,6249 \\
\hline & La Poma & Cabo la Poma & Cereal & 2 & & $3 .^{a}$ & 0,143 & 0,143 & 225 & 0,4352 \\
\hline & Viadós & Viadós & Cereal & 4 & 6 & $3 .^{a}$ & 0,322 & 0,3935 & 28 & 0,5126 \\
\hline & & & Prado & 1 & & $3 .^{a}$ & 0,0715 & & & \\
\hline & Viadós & Viadós & Prado & 4 & & $3 .^{a}$ & 0,286 & 0,286 & 22 & 0,3767 \\
\hline & Viadós & Ballariza & Inculto & 2 & & & 0,143 & 0,143 & 77 & 0,066 \\
\hline & & & & 21 & 6 & & 1,5375 & 1,5375 & & 2,0154 \\
\hline \multicolumn{11}{|l|}{ Añada Viciele } \\
\hline & Igüerra & Cabo Igüerra & Cereal & 6 & & $2 .^{a}$ & 0,429 & 1,025 & 238 & 1,5618 \\
\hline & & & Cereal & 8 & 4 & $3 .^{a}$ & 0,596 & & & \\
\hline & Igüerra & La Cruz & Cereal & 8 & & $3 .^{a}$ & 0,572 & 0,57218 & $31+182$ & 0,2952 \\
\hline & Viciele & Cabo de Viciele & Cereal & 1 & 4 & $3 .^{a}$ & 0,0955 & 0,0955 & 107 & 0,099 \\
\hline & & & & 23 & 8 & & 1,6925 & 1,6925 & & 1,956 \\
\hline \multicolumn{11}{|c|}{ Casa Cabo: 7 parcelas en propiedad, 2 en panares } \\
\hline \multirow[t]{2}{*}{ Añada La Poma } & La Poma & Cabo la Poma & Cereal & 2 & & $3 .^{a}$ & 0,143 & 0,143 & 206 & 0,817 \\
\hline & & & & 2 & 0 & & 0,143 & 0,143 & & 0,817 \\
\hline \multirow[t]{2}{*}{ Añada Viciele } & Igüerra & Cabo Igüerra & Cereal & 1 & 2 & $3 .^{a}$ & 0,0835 & 0,0835 & 240 & 0,291 \\
\hline & & & & 1 & 2 & & 0,0835 & 0,0835 & & 0,291 \\
\hline
\end{tabular}


Panar Partida Cultivo Superficie Calidad Superficie (ha) Parc. Sup.

actual real

\begin{tabular}{|c|c|c|c|c|c|c|c|c|c|c|}
\hline \multirow[t]{2}{*}{ Añada La Poma } & \multirow[t]{2}{*}{ Viadós } & \multirow[t]{2}{*}{ Viadós } & \multirow[t]{2}{*}{ Cereal } & 1 & & \multirow[t]{2}{*}{$3 .^{a}$} & 0,0715 & 0,0715 & \multirow[t]{2}{*}{30} & 0,1972 \\
\hline & & & & 1 & 0 & & 0,0715 & 0,0715 & & 0,1972 \\
\hline Añada Viciele & & & & 0 & 0 & & 0 & 0 & & 0 \\
\hline
\end{tabular}

Se anotan la parcela actual correspondiente y la superficie según el catastro actual.

Nota: en 1863 había en Gistaín 84 propietarios de tierras. Los ejemplos presentados abarcan un abanico de casas con patrimonio medio-alto a medio-bajo. La media de parcelas por casa era 13. Sólo una casa tenía más de 30 parcelas, mientras 15 disponían de entre 20 y 30. Entre las casas más pobres, 13 propietarios tenían 5 o menos fincas.

Fuente: elaboración propia a partir del amillaramiento del municipio de Gistaín.

\section{ALGUNOS APUNTES SOBRE EL ORIGENY EVOLUCIÓN DE LOS PANARES DE GISTAÍN}

Algunos documentos antiguos conservados en el archivo municipal de Gistaín hacen referencia a sus panares. En un documento fechado en $1555^{32}$ se alude a los panares de Viadós y de Lisiert, por lo que sabemos que para esta fecha ya estaban operativos, aunque no podemos saber si las parcelas tenían consideración particular o comunal. Se trata, además, de los dos panares situados a mayor distancia del pueblo, por lo que hay que descartar la hipótesis de que los panares fueran resultado de una progresiva puesta en cultivo de espacios cada vez más alejados, ligados a la explosión demográfica del siglo XIX.

En otro documento, fechado en 1469 (Gómez de Valenzuela, 2007: 159), se habla del arriendo del término de La Poma por tres años a ganaderos foráneos, de Berbegal, por un precio de 847 sueldos anuales. Esto nos indica que La Poma se destinaba entonces a pastos estivales y no a cultivos de centeno, por lo que la constitución del panar de La Poma podría ser posterior a esta fecha. Aunque podría ser también que los cultivos existieran con anterioridad y se hubieran abandonado debido a las condiciones climáticas. Entre finales del siglo XV y mediados del XVI fueron frecuentes los brotes de peste y las enfermedades del ganado. Las décadas de 1430 y 1480 estuvieron marcadas por fríos intensos (Pascua, 2012: 94), lo que pudo provocar el abandono circunstancial de los cultivos en altitud y su aprovechamiento entonces como pastos.

Los estudios paleoambientales realizados a partir de la secuencia polínica de la Basa de la Mora, en el mismo valle de Gistaín, sugieren una intensificación de la actividad hu-

32.Archivo del Ayuntamiento de Gistaín, caja 2/32. «Vendición de las partidas de Orieles, El Forcallo y Fontaciellas al Ayuntamiento de Gistaín por parte de Felipe de Bardají, Señor de San Juan». 
mana y su huella sobre el paisaje a partir del siglo XIV. En este periodo un claro aumento en el registro de polen de fresno parece indicar que en él hay que situar el origen del sistema prado de siega-borda (Pérez Sanz et al., 2011), posiblemente al tiempo que el de los panares. El enfriamiento considerable a partir del año 1600 aproximadamente dificultaría mucho los cultivos en altitud. Por ejemplo, las parcelas de cereal situadas en El Chelau (1900 m), que, como su nombre indica, es una zona muy fría aún en época actual, podrían ser una ocupación posterior, relacionada con el paulatino aumento de la temperatura a partir de finales del siglo XIX y al mismo tiempo con el incremento de la población y las mayores necesidades de manutención humana.

Un indicador de esta expansión en las cotas más altas es el registro de parcelas en estas áreas de los panares. El amillaramiento de 1863 no refleja prácticamente parcelas en partidas como La Fesa, que, aunque estén ahora abandonadas, son recordadas por los habitantes actuales ${ }^{33}$. Igual ocurre en partidas como El Chelau, La Coda o Cabo Viciele, donde el número de parcelas en 1863 es inferior al que podemos observar en el catastro actual, teniendo en cuenta que muchos de los campos aún reconocibles y ratificados como cultivos de cereal por el testimonio oral de los habitantes no están registrados en el catastro. Todo esto señala un claro aumento de la superficie cultivada en las cotas más altas de los panares en las últimas décadas del siglo XIX y la primera parte del Xx, que vemos reflejado en el número de parcelas considerado dentro de los panares en los Cuadros 2 y 3 . No podemos descartar que, en fases cálidas anteriores, estos espacios ya fueran puestos en cultivo. En el caso de Gistaín, la tradición oral sitúa la población primigenia en Las Fuens, en la franja superior del panar de La Poma, aproximadamente a $1850 \mathrm{~m} \mathrm{s.} \mathrm{n.} \mathrm{m.}$ Por el momento nada se sabe de este posible asentamiento, ni de su carácter permanente o temporal, pero nos sugiere una ocupación del espacio en altitud muy diferente de la actual.

Al tiempo que los panares se expandían en las cotas más altas, ya en contacto con los pastos comunales, aparecieron en ellos, ya entrado el siglo xx, parcelas dedicadas a prados, cerradas con muro de piedra seca para negar el paso a los rebaños comunales de ovino. Tenemos el caso del cerrado de Casa Ferrer $\left(0^{\circ} 19^{\prime} 14,154\right.$ ' E $42^{\circ} 36^{\prime} 28,911^{\prime \prime N}$; 1810 m s. n. m.) en la parte alta del panar de La Poma. El mismo fenómeno se da en el conjunto de cercados de la partida de Macarrons $\left(0^{\circ} 18^{\prime} 18,434^{\prime \prime} \mathrm{E} 42^{\circ} 36^{\prime} 43,039^{\prime \prime} \mathrm{N}\right.$; 1800-1900 m s. n. m.), muy próxima, pero ya en el término municipal de Serveto. Según recogió Joaquín Villa (1986), en los panares vedados no se podía ni siquiera segar

33. Esta partida parece haber sido puesta en cultivo tardíamente, en terrenos ganados al bosque, y cultivada durante pocas décadas, en condiciones difíciles de accesibilidad. No aparece en el catastro actual. 
la hierba que crecía en los márgenes de la parcela. Pero si una finca se dedicaba a prado, y se construía una borda para el almacenamiento de la hierba, era posible amojonarla para segregarla del espacio pastado comunalmente. Esto podría añadir un elemento de explicación a la existencia de tan alto número de bordas en el municipio. Todo aquel que se podía permitir su construcción lo hacía, y de esta forma podía soslayar la imposición comunal.

El declive de los panares se produjo a partir de la década de los sesenta del siglo Xx. La tradición oral sitúa la cifra de ovinos en unos 12.000 antes de la Guerra Civil ${ }^{34}$, y aún se mantenía sobre los 5.000 en los primeros años de posguerra. Pero la llegada de las comunicaciones viarias propició la emigración masiva, con el consiguiente desmoronamiento del sistema económico tradicional desarrollado desde la Baja Edad Media. A partir de ese momento se produjo el cambio de modelo ganadero, que se orientó hacia el vacuno. La entrada de cereales asequibles importados de fuera del valle hizo innecesarios los cultivos en los panares $^{35}$, por otra parte ya inviables por falta de mano de obra y del abonado que proporcionaban las ovejas.

\section{DISCUSIÓNY CONCLUSIONES}

Los panares constituyen un interesante ejemplo de organización comunal para la explotación de unos recursos escasos y dispersos, de modo que se pudiera extraer el máximo aprovechamiento de unas tierras fuertemente condicionadas por el clima y el relieve.

Por un lado, los panares supusieron una ampliación de la superficie agraria útil, pero, al mismo tiempo, una salvaguarda del espacio de pastos necesario para la creciente cabaña ovina trashumante. La existencia de estos campos de cereal suponía una competencia para la ganadería ovina, que durante siglos fue la principal fuente de ingresos en los altos valles del Pirineo aragonés. Por ello, el ciclo agrícola se adaptaba y complementaba el ciclo ganadero.

El origen del sistema parece estar en la Baja Edad Media, momento en que se produjo una expansión demográfica -no sin altibajos debido a las pestes y a anomalías climáticas-y en el que se desarrolló la trashumancia a gran escala de rebaños de ovejas hacia la depresión del Ebro. Desconocemos su evolución en los siglos siguientes. En un

34.Es la misma cifra que da Soler i Santaló hacia 1910 (1916).

35. Con el importe de la venta de un solo cordero una familia se podía abastecer de cereal todo el año. Información oral recogida en San Juan de Plan. 
principio pudieron ser aprovechamientos particulares sobre suelo comunal, para pasar después a la propiedad de las parcelas con una serie de restricciones regladas para el aprovechamiento comunal de los rastrojos tras la cosecha. Se abre aquí una línea de investigación que podría situar a los panares en el debate en torno a los comunales (Beltran \& Vaccaro, 2017), relacionándolos con otras formas intermedias de propiedad desarrolladas en las áreas de montaña, como serían los panificados y los quiñones en áreas navarras (Alli, 1989), las artigas o los ampriús. También otras formas de ocupación ganadera que podían albergar al mismo tiempo aprovechamientos agrícolas y que combinaban elementos de propiedad privada y comunal (Corbera, 2013).

La evolución de los panares ha podido estar condicionada por circunstancias climáticas y por cambios socioeconómicos, pero pervivieron como forma de organización durante varios siglos y en algunos casos hasta mediados de siglo $\mathrm{xx}$, lo que demuestra su validez en un sistema económico complejo en el que muchos elementos interactuaban íntimamente ligados entre sí (Moreno, 2002). Su interpretación es imposible sin tener en cuenta su interacción con la trashumancia. Su impronta sigue presente, aun perdido su uso original y tras transformarse en prados o en pastos a diente, dentro de un paisaje humanizado dinámico y cambiante a lo largo del tiempo que se acompasa a las variaciones de contexto, tanto climático como humano.

Por otro lado, no en todos los valles del Pirineo central se desarrolló este sistema. Las razones por las que se encuentra en unos y no en otros no están del todo claras. Se han apuntado algunas ideas, relacionadas con la configuración geográfica de los valles o las diferencias climáticas entre los valles occidentales y los orientales, pero habría que estudiar en profundidad más localizaciones para tener un diagnóstico más ajustado.

También se encuentran notables diferencias entre unos y otros panares. En el caso de Gistaín, son varios los que se disponen comprimidos entre áreas de pastos comunales y las parcelas del fondo de valle en régimen de propiedad, en las que coexistían los prados con el cereal, y que desembocaron en un esquema de bocage exclusivamente de prados. Otros aparecen en áreas mucho más alejadas y completamente rodeados de comunal, como es el caso de Viadós.

Los panares estudiados en Gistaín se caracterizaron por un elevado número de construcciones accesorias en las parcelas que lo componían, como cabañas en la mayor parte de ellas, muchas con corral de ordeño asociado, y también bordas. Esta abundancia de construcciones no existe en muchos panares de otras localidades, hecho que puede estar relacionado con la organización particular en Gistaín de los rebaños en los periodos anterior y posterior a la estancia en los pastos de verano. 
Con todo, uno de los aspectos más olvidados es el de la organización del uso de estos espacios. Las normas que regían el régimen de alternancias en el cultivo, las fechas de veda y desveda, el ámbito supeditado o no a la regulación, o la composición de las añadas en núcleos con elevado número de panares dispersos en su territorio son temas aún por estudiar. Con la urgencia, además, de hacerlo antes de que desaparezcan los últimos habitantes que puedan aportar esta información, en los valles en los que aún se recuerde, dado el carácter consuetudinario de estas normas.

\section{AGRADECIMIENTOS}

A los evaluadores anónimos de la revista Historia Agraria, por sus comentarios y sugerencias al primer borrador y especialmente a Enric Saguer, por sus correcciones y comentarios, que ayudaron a mejorar muy notablemente el texto final.

A Joaquín Quino Villa, Fernando Biarge, Federico Fillat y a los habitantes de Gistaín, a partir de cuyas aportaciones se ha conformado en gran parte este trabajo.

\section{REFERENCIAS}

ABAD, F. (2016). La implantación de la patata en Aragón. Temas de Antropología Aragonesa, (22), 93-102.

Alli, J. C. (1989). La mancomunidad del Valle del Roncal. Pamplona: Gobierno de Navarra.

AzCÁRATe, M.V. (1990). El viñedo en El Sobrarbe durante el siglo XI: Su localización. Espacio, tiempo y forma. Serie VI, Geografía, (3), 151-166.

Balcells, E. \& Creus-Novau, J. (1986). Reflexiones sobre los límites altitudinales de las residencias humanas permanentes en el Alto Pirineo aragonés. Pirineos, (127), 153174. https://digital.csic.es/handle/10261/95397

BALlarín, A. (1968). El valle de Benasque: Formación, pasado, presente y porvenir del valle. Zaragoza: La Editorial.

Beltran, O. \& Vaccaro, I. (2017). Los comunales en el Pirineo Central: Idealizando el pasado y reelaborando el presente. Revista de Antropología Social, 26 (2), 235-257. https://doi.org/10.5209/RASO.57605

Biarge, F. (2009). Sobrarbe: Letra menuda. Huesca: Diputación Provincial de Huesca. Biarge, F. \& Biarge, A. (2000). Piedra sobre piedra: El paisaje pirenaico humanizado. Huesca: F. Biarge/A. Biarge. 
Bielza de Ory, V., Corral, J. L., Escolano, S., Laliena, C., Sesma, A. \& Ubieto, A. (1986). Estudio histórico-geográfico del valle de Bielsa, Huesca. Huesca: Instituto de Estudios Altoaragoneses/Diputación Provincial de Huesca.

Blas, F. \& Romanos, F. (2008). Diccionario aragonés chistabin-castellano (Bal de Chistau). Zaragoza: Gara d'Edizions.

BuIL, L. J. (1997). Viaje por el Alto Aragón, noviembre del año 1794. Huesca: La Val de Onsera.

Buisán, L. (2004). La Solana de Burgasé y la Ribera de fánovas: Nostalgia de un paisaje. Huesca: L. Buisán.

Cavailllès, H. (1931). La vie pastorale et agricole dans les Pyrénées des Gaves, de l'Adour et des Nestes: Étude de géographie humaine. Paris: Armand Colin.

Chevalier, M. (1956). La vie humaine dans les Pyrénées ariégeoises. Paris: M.-Th. Génin, Librairie de Médicis.

Comas, D. \& Pujadas, J. J. (1994). Estudios de antropología social en el Pirineo aragonés. Zaragoza: Gobierno de Aragón.

COMITÉ INTERGOUVERNEMENTAL POUR LA PROTECTION DU PATRIMOINE MONDIAL CULTUREL ET NATUREL (2008). Orientations devant guider la mise en oeuvre de la Convention du patrimoine mondial. Paris: UNESCO.

Corbera, M. (2013). Organización de los espacios de pastos en la montaña atlántica: Los nombres, las formas y las funciones. Ería, (92), 275-292. https://www.unioviedo.es/ reunido/index.php/RCG/article/view/10141/0

Cuesta, J. M. (2001). La despoblación del Sobrarbe: ¿Crisis demográfica o regulación? Zaragoza: Centro de Estudios sobre la Despoblación y Desarrollo de Áreas Rurales.

Daumas, M. (1961). Le régime pastoral du Haut-Aragon oriental. Études Rurales, (3), 5-20. https://doi.org/10.3406/rural.1961.984

Daumas, M. (1976). La vie rurale dans le Haut Aragon oriental. Madrid: Institutos de Estudios Oscenses y de Geografia Aplicada.

FiLlAT, F. (1980a). La explotación ganadera como estrategia para frenar la pérdida de fertilidad en montaña. Cuadernos de Investigación Geográfica, (6), 37-50. https://digital.csic.es/handle/10261/59407

FiLlat, F. (1980b). Explotación del Alto Aragón por la población humana: Explotación indirecta (ganadería). En Actas del I Congreso Español de Antropología (pp. 67-76). Vol. 1. Barcelona, 28 de marzo-2 de abril de 1977. http://digital.csic.es/handle/10261/ 104218

FILlat, F. (2003). Un paisaje pirenaico de prados y pastos: Cambios recientes y perspectivas. Acta Botanica Barcinonensia, (49), 313-324. http://www.raco.cat/index.php/ ActaBotanica/article/view/59857/99364 
Fillat, F., Gómez García, D. \& Creus-Novau, J. (1984). El fresno de hoja ancha como árbol semi-salvaje en el Pirineo de Huesca (Aragón). Acta Biologica Montana, (4), 445454. http://digital.csic.es/handle/10261/108390

FiLlat, F. \& Villar, L. (1983). Paisajes vegetales y ganadería en la alta cuenca del Gállego. Facetania, (102), 27-31. https://digital.csic.es/handle/10261/53338

García Ruiz, J. M. (1976). Modos de vida y niveles de renta en el Prepirineo del Alto Aragón Occidental. Jaca: Consejo Superior de Investigaciones Cientificas/Instituto de Estudios Pirenaicos. http://digital.csic.es/handle/10261/96875

GARCÍA RuIZ, J. M. (1988). La evolución de la agricultura de montaña y sus efectos sobre la dinámica del paisaje. Revista de Estudios Agrosociales, (146), 7-37. http://hdl.handle.net/10261/100832

García Ruiz, J. M., López Moreno, J. I., Lasanta, T., Vicente, S. M., González SaMpériz, P., Valero, B. L., Sanjuán, Y., Beguería, S., Nadal, E., Lana, N. \& Gómez VILLAR, A. (2015). Los efectos geoecológicos del cambio global en el Pirineo central español: Una revisión a distintas escalas espaciales y temporales. Pirineos, (170). e012. https://doi.org/10.3989/Pirineos.2015.170005

Gómez de ValenZuela, M. (2003). La vida en el valle de Tena en el siglo XVI. Huesca: Instituto de Estudios Altoaragoneses/Ayuntamiento de Sallent de Gállego.

GómeZ DE VALENZUELA, M. (2007). Documentos sobre ganadería altoaragonesa y pirenaica (siglos $X V$ y XVI). Zaragoza: El Justicia de Aragón.

González Samperiz, P., Aranbarri, J., Pérez Sanz, A., Gil Romera, G., Moreno, A., Leunda, M., Sevilla, M., Corella, J., Morellón, M., Oliva, B. \& Valero, B. (2016). Environmental and Climate Change in the Southern Central Pyrenees since the Last Glacial Maximum: A View from the Lake Records. Catena, (149), 668-688.

KuLA, W. (1980). Las medidas y los hombres.Madrid: Siglo XXI de España.

LASANTA, T. (1989). Evolución reciente de la agricultura de montaña: El Pirineo Aragonés. Logroño: Geoforma.

Lasanta, T., García Ruíz, J. M. \& OrTigosa, L. M. (1994) Distribución espacial de diferentes modelos de campos de cultivos en el Pirineo central español. Ería, (133), 6372. http://hdl.handle.net/10261/100830

Lasaosa, R. \& Ortega, M. (2003). Miradas desde Tella. Huesca: Ayuntamiento de Tella-Sin/La Val de Onsera.

Monserrat, P. (1992). La gestión ecológico-cultural en el paisaje de montaña. Pirineos, (140), 53-73. https://doi.org/10.3989/pirineos.1992.v140.172

Monserrat, P. (2009). La cultura que hace el paisaje. Estella: La Fertilidad de la Tierra. Monserrat, P. (2014). Ecología del paisaje humanizado. En VII Cursillo sobre Flora y Vegetación en el Pirineo. Jaca, 16-20 de julio de 2002. http://digital.csic.es/handle/10261/100001 
Monserrat, P. \& Villar, L. (1995). Los agroecosistemas. En L. Villar (Ed.), Historia natural'93 : Actas de la XI Bienal de la Real Sociedad Espanola de Historia Natural (pp. 157-168). Jaca, 13-18 de septiembre de 1993. Jaca: Instituto de Estudios Altoaragoneses/Instituto Pirenaico de Ecología. http://digital.csic.es/handle/10261/54409

Moreno, J. R. (2002). La economía de montaña en el Antiguo Régimen: Los equilibrios tradicionales en el Pirineo aragonés. Ager, (2), 43-80. https://recyt.fecyt.es/index.php/ AGER/article/view/7023

Pallaruelo, S. (1993). Pirineo aragonés. Madrid: Instituto Nacional para la Conservación de la Naturaleza. (Cuadernos de la trashumancia, 6).

Pardinilla, I. (2013). Estudio de los paisajes culturales del bien Pirineos-Monte Perdido. En «Estudios sobre el patrimonio cultural del bien Pirineos-Monte Perdido inscrito en la Lista del Patrimonio Mundial». Documento inédito.

Pascua, E. (2012). Señores del paisaje: Ganadería y recursos naturales en Aragón, siglos XIII-XVII. Valencia: Universitat de València.

Pérez Sanz, A., González Sampériz, P., Valero, B. L., Moreno, A., Morellón, M., Sancho, C., Belmonte, A., Gil-Romera, G., Sevilla, M. \& Navas, A. (2011). Clima y actividades humanas en la dinamica de la vegetacion durante los últimos 2000 años en el Pirineo central: El registro palinologico de la Basa de la Mora (Macizo de Cotiella). Zubia, (23), 17-38. http://hdl.handle.net/10261/47592

RuAs, M. P., RENDU, C. \& BERgERET, A. (2005). Glanes et cultures médiévales en haute montagne d'après les restes de graines et de fruits carbonisés de deux sites médiévaux de Cerdagne et du Capcir (Pyrénées-Orientales). En A. CATAFAu (Ed.), Les ressources naturelles des Pyrénées du moyen âge à l'époque moderne: Exploitation, gestion, appropriation: Actes du Congrès International RESOPYR 1 (pp. 147-184). Font-Romeu, 8-10 de noviembre de 2002. Perpignan: Presses Universitaires de Perpignan.

Soler i Santaló, J. (1916). La vall de Gístaín. Butlletí del Centre Excursionista de Catalunya, (258-259-260).

Utrilla, J. F., Laliena, C. \& NAVARRo, G. (2005). Los recursos naturales y su transformación en los Pirineos aragoneses durante la Edad Media. En A. Catafau (Ed.), Les ressources naturelles des Pyrénées du moyen âge à l'époque moderne: Exploitation, gestion, appropriation: Actes du Congrès International RESOPYR 1 (pp. 19-48). Font-Romeu, 8-10 de noviembre de 2002. Perpignan: Presses Universitaires de Perpignan.

VILLA, J. (1986). «Chistén: Un pueblo de pastores». Documento inédito. 